\title{
Antifungal effects of Rhus coriaria $L$. fruit extracts against tomato anthracnose caused by Colletotrichum acutatum
}

\begin{abstract}
The prevalence of Anthracnose disease, which is caused by a number of Colletotrichum spp., takes its toll on the tomato (Lycopersicon esculentum Mill.) and poses a significant economic constraint on its production. Successful efforts towards controlling this organism remain a deficient task. Rhus coriaria fruit extracts were subjected to screening, in vitro and in vivo, for antifungal activities. Under light and Scanning Electron Microscopy (SEM), the crude extractôs effect on the morphological structures of hyphae underwent an observation and study. Experimental results surfaced proofs that acetone, aqueous, methanol, and ethanol extracts have fungicidal properties on C. acutatum (GenBank accession No. KT215296) growth, which multiplied when extract concentrations were higher. Observations from scanning electron microscope showed that active extracts led to hyphal degradation where the hyphae were retarded. Tomato plants and fruits, which were treated with aqueous extracts, have been observed to have a significantly lower disease incidence, index, and severity. The anthracnose incidence in fruits that were coated with aqueous extract exhibited a maximum reduction with severity scores consistently at $(0 \%)$. Aqueous extract in the glass house test significantly reduced the total area under the disease progress curve (AUDPC) and disease severity in the studied disease. The shoot height, root length, dry shoot height, dry root length, chlorophyll content and leaf surface area significantly improved and increased with these treatments. The results indicate that $\mathrm{R}$. coriaria extract can be judged as a cost effective and eco-friendly replacement to chemical fungicides in the management of tomato anthracnose disease.
\end{abstract}

Keyword: Anthracnose; Sumac; Colletotrichum sp.; Biological control; AUDPC; SEM 